\title{
BMJ Open Developing and validating a model for predicting 7-day mortality of patients admitted from the emergency department: an initial alarm score by a prospective prediction model study
}

Ming-Ju Hsieh, ${ }^{1}$ Nin-Chieh Hsu (D) , ${ }^{2,3}$ Yu-Feng Lin, ${ }^{2,3}$ Chin-Chung Shu (D) ,,3 Wen-Chu Chiang, ${ }^{4}$ Matthew Huei-Ming Ma, ${ }^{5}$ Wang-Huei Sheng ${ }^{3}$

To cite: Hsieh M-J, Hsu N$\mathrm{C}$, Lin Y-F, et al. Developing and validating a model for predicting 7-day mortality of patients admitted from the emergency department: an initial alarm score by a prospective prediction model study. BMJ Open 2021;11:e040837. doi:10.1136/ bmjopen-2020-040837

- Additional material is published online only. To view please visit the journal online (http://dx.doi.org/10.1136/ bmjopen-2020-040837).

$\mathrm{N}-\mathrm{CH}$ and C-CS contributed equally.

Received 25 May 2020

Revised 17 November 2020 Accepted 23 November 2020

Check for updates

(C) Author(s) (or their employer(s)) 2021. Re-use permitted under CC BY-NC. No commercial re-use. See rights and permissions. Published by BMJ.

For numbered affiliations see end of article.

\section{Correspondence to}

Dr Chin-Chung Shu; ccshu139@ntu.edu.tw and Dr Nin-Chieh Hsu;

chesthsu@gmail.com

\section{ABSTRACT}

Objectives To set up a prediction model for the 7-day inhospital mortality of patients admitted from the emergency department $(E D)$ because it is high but no appropriate initial alarm score is available.

Design This is a prospective cohort study for prediction model development.

Setting In a tertiary referred hospital in northern Taiwan. Participants ED-admitted medical patients in hospitalist care wards were enrolled during May 2010 to October 2016. Two-thirds of them were randomly assigned to a derivation cohort for development of the model and crossvalidation was performed in the validation cohort.

Primary outcome measured 7-day in-hospital mortality. Results During the study period, 8649 patients were enrolled for analysis. The mean age was 71.05 years, and $51.91 \%$ were male. The most common admission diagnoses were pneumonia (36\%) and urinary tract infection (20.05\%). In the derivation cohort, multivariable Cox proportional hazard regression revealed that a low Barthel Index Score, triage level 1 at the ED, presence of cancer, metastasis and admission diagnoses of pneumonia and sepsis were independently associated with 7 days in-hospital mortality. Based on the probability developed from the multivariable model, the area under the receiver operating characteristic curve in the derivation group was $0.81(0.79-0.85)$. The result in the validation cohort was comparable. The prediction score modified by the six independent factors had high sensitivity of $88.03 \%$ and a negative predictive value of $99.51 \%$ for a cut-off value of 4 , whereas the specificity and positive predictive value were $89.61 \%$ and $10.55 \%$, respectively, when the cut-off value was a score of 6 .

Conclusion The 7-day in-hospital mortality in the hospitalist care ward is $2.8 \%$. The initial alarm score could help clinicians to prioritise or exclude patients who need urgent and intensive care.

\section{INTRODUCTION}

In-hospital mortality is a universally accepted indicator for the medical quality of inpatient care. ${ }^{1}$ Although mortality is usually very
Strengths and limitations of this study

- The six-factor prediction score was based on a large-scale cohort using cross-validation methods.

- The prediction score for 7-day in-hospital mortality could be used before vital signs changed and focus on the subacute phase (2-7 days) after hospitalisation instead of the 24 hours mortality predicted by the National Early Warning Score.

- The data of vital signs have not been incorporated into the model.

- The results have not been proved to be generalisable to other ethnic groups and specialty care models.

low for elective admission, it is comparably higher for admissions from the emergency department (ED). ${ }^{2}{ }^{3}$ The 30-day mortality of emergency admission has been reported to be $3.7 \%-8.2 \%$ in a report from Ireland, ${ }^{4}$ and $4.7 \%-5.8 \%$ and $6.93 \%-7.04 \%$ in two UK reports, respectively. ${ }^{56}$ Notably, the risk of death is relatively high in the initial days from emergency stay to hospitalisation. ${ }^{4}$ Therefore, identifying patients at risk of deterioration during their initial hospitalisation days is a great challenge but important to clinicians.

One commonly used prediction score is the National Early Warning Score (NEWS), which is mainly composed of vital signs and consciousness level. However, changes in vital signs can indicate acute changes in the illness leading to a possibly irreversible late stage, and the NEWS is suggested for only very short prognosis, such as within 24 hours. ${ }^{7}$ However, there are scarce studies reported mortality prediction model for subacute phase (2-7 days) after hospitalisation. It is particularly crucial to predict in-hospital mortality within 7 days at the initial admission because emergency admissions have been reported to 
have high in-hospital mortality of $6.2 \%-12 \%$ in previous reports, because deterioration is not uncommon. ${ }^{89} \mathrm{In}$ addition, for in-hospital prognosis before vital signs change, an initial predictive score that prioritises the inpatient's care is needed for admission evaluation. Therefore, in this study, we conducted a prospective observational study in a hospitalist model for emergency-admitted patient care in Taiwan and developed a prediction model for 7-day in-hospital mortality using available information at admission, including patient demographics, admission diagnosis, underlying comorbidities and performance.

\section{METHODS}

\section{Source of data}

We prospectively conducted a 6-year hospital-based cohort study in a tertiary referral medical centre in Taipei, Taiwan. Participants were limited to adult $(\geq 20$ years old) non-trauma patients admitted from the ED to hospitalist care general wards. We did not include patients needing surgery or subspecialty care such as care for HIV infection or chemotherapy for haematological or oncology diagnosis. The assignment of patient admission is decideded by ED physician, not by hospitalists, accordingly to aforementioned rules. We consecutively recruited patients from the period of 1 May 2010 to 31 October 2016. Written informed consent was waived due to the observational design. Patients transferred to other wards before discharge were excluded from the analysis of in-hospital outcomes.

The enrolled patients were divided into two groups by a computerised random process. Two-thirds of the patients were classified as a derivation cohort, and the remaining one-third were used as a validation cohort.

\section{Patient and public involvement}

Patients or the public were not involved in the design, or conduct, or reporting, or dissemination plans of our research. The study results were not disseminated to study participants.

\section{Study settings and participants}

The tertiary medical centre where our study was performed contained around 2450 beds, and 9500 patients visit the ED per month. Of these 9500 , nearly $20 \%$ are admitted. The inpatient wards had 36 beds for one ward from October 2009 to May 2013 and then 71 beds for two wards from June 2013 to October 2016, which were tended by hospitalists.

\section{Outcome and clinical characteristics}

The outcome of interest was 7-day in-hospital mortality. The in-hospital duration was defined as the time interval from ED admission to death or discharge of the patient. The demographic and clinical data, including age, sex, season of ED admission, triage level at ED arrival, underlying comorbidities, Barthel index at ward admission and admission diagnosis were coded in a standard case report form with default options by trained assistants. In our study, the Taiwan Triage and Acuity Scale (TTAS) was used in the ED. ${ }^{10}$ One medical patient would be assorted as triage level 1 if one of the following conditions happened at ED arrival: (1) cardiopulmonary arrest, (2) severe respiratory distress with oxygen saturation $<90 \%$ on the pulse oximeter, (3) signs of shock, (4) Glasgow Coma Scale $\leq 8$ points, (5) body temperature $>41^{\circ} \mathrm{C}$ and (6) the patient transferred with endotracheal tube. The patient assorted as triage level 1 got admitted to the general ward if the patient was stabilised at the ED.

\section{Statistical analysis methods}

After dividing the cases into derivation and validation cohorts, we used locally weighted least squares regression (LOWESS) curves to first find the inflection points of continuous predictors in the derivation cohort. We then transformed the continuous predictors into categorical predictors by using the inflection point on the curve. If the inflection point could not be found, we used threshold values adopted in either one or more of the existing EWSs or from clinical experience. Next, we performed univariable Cox proportional hazard regression for each predictor with the outcome. If the $p$ value of one predictor was less than 0.10 in the univariable analysis, it was put into the multivariable stepwise Cox proportional hazard regression. The area under the receiver operating characteristic curve (AUROC) was used to examine discrimination in the derivation and validation cohorts.

To make the prediction model easy to use for medical personnel, we modified the beta coefficient of each statistically significant predictor in multivariable stepwise Cox proportional hazard regression into an integer by multiplying the same but smallest number and adopting the nearest integer. The integer point value system was then built up, and the best cut-off value was chosen using Youden's Index. We then used this value to calculate the sensitivity, specificity, positive predictive value (PPV) and negative predictive value (NPV) when the model was applied separately to the derivation and validation cohorts. Then we tried to find the cut-off value with $>85 \%$ sensitivity and the highest possible specificity, and that with $>85 \%$ specificity and the highest possible sensitivity. Data were analysed in SAS software (V.9.4, SAS Institute) and R software (V.3.3.2). Statistical significance was defined as $\mathrm{p}<0.05$.

\section{RESULTS}

\section{Enrolled participants}

During the study period, a total of 9093 patients were admitted to the general ward. After excluding 444 (4.9\%) patients with incomplete data (167 missed Barthel index, 275 missed triage level and two patients missed survival status), 8924 patients were included in the final analysis. Within 7 days of hospitalisation, 275 patients were 


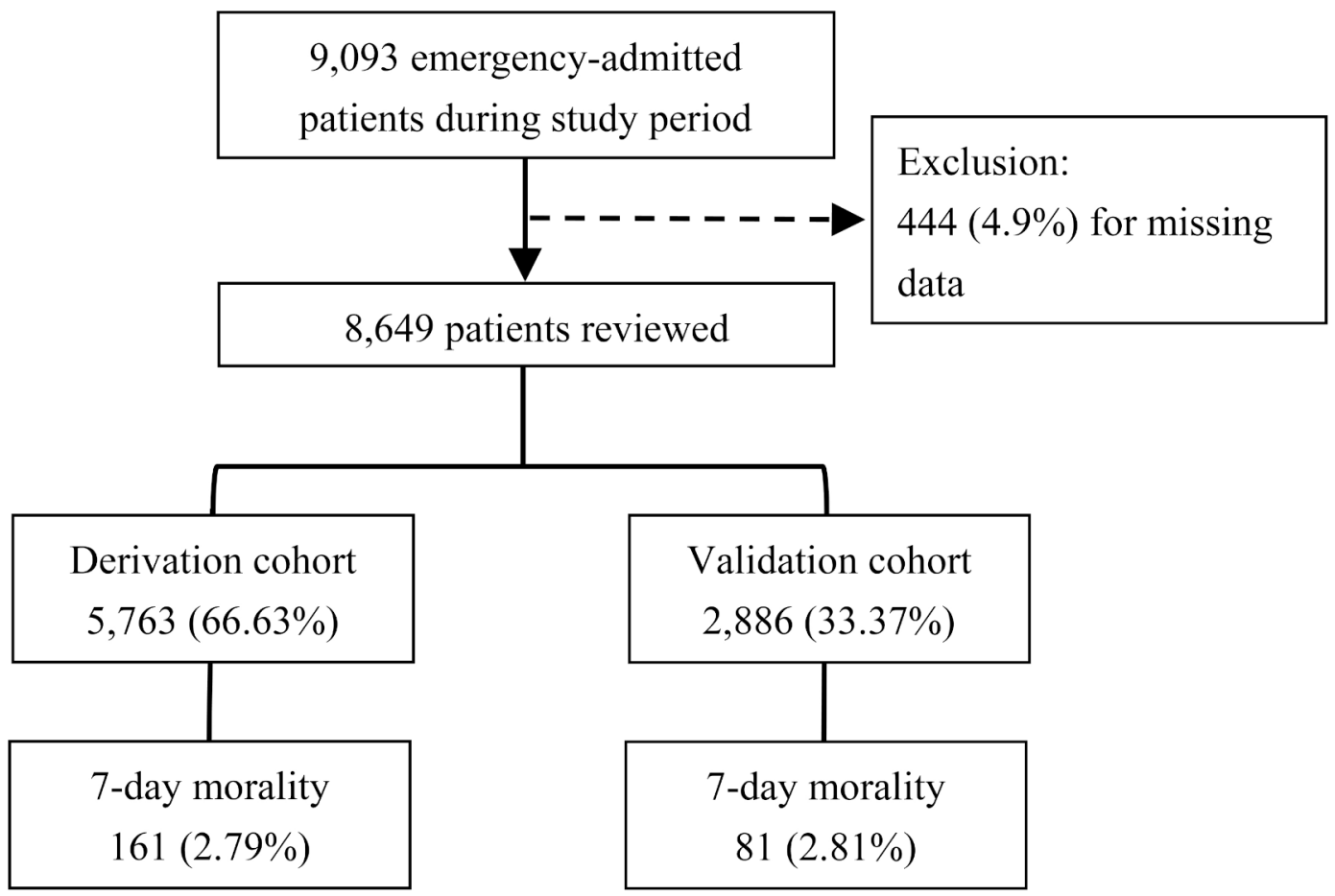

Figure 1 Flow chart of participant recruitment and outcomes.

transferred to other wards and exclude from the analysis. A flow chart of the cases is shown in figure 1 .

The characteristics of the derivation cohort, the validation cohort and all patients are shown in table 1 . The mean age of the patients was 71.05 years, and $51.91 \%$ were male. Around $10.71 \%$ of the patients had triage levels of 1 at ED, and $24.94 \%$ had active cancer. Barthel Index Scores at admission were totally dependent (scores of $<35)(30.67 \%)$ and severely dependent (scores of 35-60 score) $(24.88 \%)$. The most common admission diagnosis was pneumonia (36\%), followed by urinary tract infection $(20.05 \%)$ and upper gastrointestinal bleeding $(9.26 \%)$.

We found no significant differences in the characteristics of the derivation and validation cohorts except that admission diagnoses of acute cholecystitis and diabetic ketoacidosis were higher in the validation cohort. A total of $242(2.80 \%)$ patients died within the first 7 days of their hospital stays.

\section{Model development, specification and performance}

Using LOWESS curves, we converted values for age from continuous variables to binary variables by using the inflection point on the curve. Univariable and multivariable Cox proportional analyses are shown in table 2. In univariable Cox analysis, age ( $\geq 80$ vs $<80$ years), Barthel Index Score ( $<35$ vs $35-60$ vs. $>60)$, season (winter vs other seasons), triage level (level 1 vs other levels), cancer, metastasis and admission diagnoses of pneumonia, bronchiectasis and sepsis were found to be associated with 7-day in-hospital mortality. The factors with $\mathrm{p}>0.10$ in unvariable analysis were listed in online supplemental table S1. Multivariable stepwise Cox proportional hazard regression revealed that Barthel Index Score, triage level at ED, presence of cancer, metastasis and admission diagnoses of pneumonia and sepsis were independently associated with 7-day in-hospital mortality. Based on the probability developed from the multivariable model, the area under the ROC curve in the derivation group was 0.81 (95\% CI 0.79 to 0.85 ) (figure 2).

After multiplication by 2 and adoption of the nearest whole number as the point value, the final model was developed (table 3). The sensitivities and specificities of each score are shown in online supplemental figure S1. In brief, using Youden's index, we determined the optimal cut-off values for the model. The sensitivity, specificity, PPV and NPV for the derivation cohort were $88.03 \%, 58.81 \%, 4.91 \%$ and $99.51 \%$, respectively, when a score of 4 was determined as the optimal cut-off value (online supplemental table S2). Similarly, we found that a cut-off value of 4 had $>85 \%$ sensitivity and the highest possible specificity. On the other hand, a score of 6 had $>85 \%$ specificity and the highest possible sensitivity (online supplemental table S2). The sensitivity, specificity, PPV and NPV were $50.69 \%, 89.61 \%, 10.55 \%$ and $98.69 \%$ when the cut-off value was 6 . Then we divided the derivation cohort into three groups according to score ranges of $0 \leq$ score $<4,4 \leq$ score $<6$ and $\geq 6$. The Kaplan-Meier survival curve showed significant differences between any two of the three groups (all $\mathrm{p}<0.001$ ) (figure 3 ).

In the validation cohort, the area under the ROC curve was 0.78 (95\% CI 0.73 to 0.82 ) (figure 2). Using the optimal cut-off value of 4 according to Youden's index, the sensitivity, specificity, PPV and NPV were $87.06 \%$, $57.24 \%, 4.35 \%$ and $99.50 \%$, respectively (online supplemental table S3), which were similar to those for the derivation cohort. 
Open access

Table 1 Characteristics of study participants

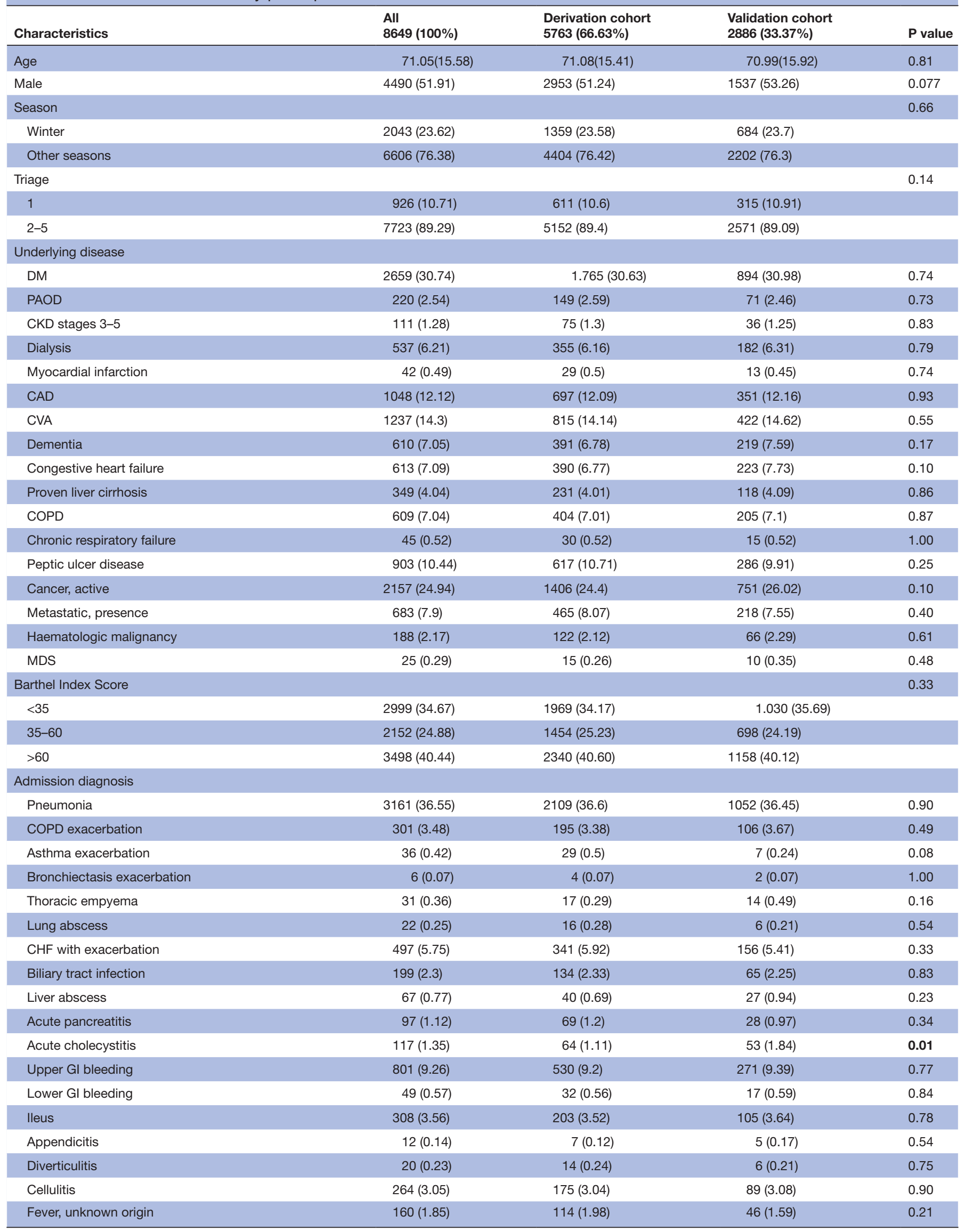


Table 1 Continued

\begin{tabular}{|c|c|c|c|c|}
\hline Characteristics & $\begin{array}{l}\text { All } \\
8649(100 \%)\end{array}$ & $\begin{array}{l}\text { Derivation cohort } \\
5763(66.63 \%)\end{array}$ & $\begin{array}{l}\text { Validation cohort } \\
2886(33.37 \%)\end{array}$ & $P$ value \\
\hline Wound infection & $46(0.53)$ & $30(0.52)$ & $16(0.55)$ & 0.84 \\
\hline Bacteremia & $531(6.14)$ & $347(6.02)$ & $184(6.38)$ & 0.52 \\
\hline Sepsis & $178(2.06)$ & $123(2.13)$ & $55(1.91)$ & 0.48 \\
\hline Ischaemic stroke & $206(2.38)$ & $142(2.46)$ & $64(2.22)$ & 0.48 \\
\hline Meningitis & $58(0.67)$ & $44(0.76)$ & $14(0.49)$ & 0.13 \\
\hline Febrile neutropenia & $123(1.42)$ & $79(1.37)$ & $44(1.52)$ & 0.57 \\
\hline Diabetic ketoacidosis & $20(0.23)$ & $9(0.16)$ & $11(0.38)$ & 0.04 \\
\hline Urinary tract infection & $1734(20.05)$ & $1143(19.83)$ & $591(20.48)$ & 0.48 \\
\hline In-hospital mortality on 7 days & $242(2.80)$ & $161(2.79)$ & $81(2.81)$ & 0.97 \\
\hline
\end{tabular}

The data are represented as number (per cent) or mean (SD) and compared with $\chi^{2}$ test or t-test, respectively.

$\mathrm{CAD}$, coronary artery disease; CHF, congestive heart failure; CKD, chronic kidney disease; COPD, chronic obstructive pulmonary disease; CVA, cerebral vascular accident; DM, diabetes mellitus; Gl, gastrointestinal; MDS, myelodysplatic syndrome; PAOD, peripheral arterial occlusive disease.

Table 2 Univariable and multivariable analyses for predictive factors of 7-day in-hospital mortality in the derivation cohort using stepwise model selection

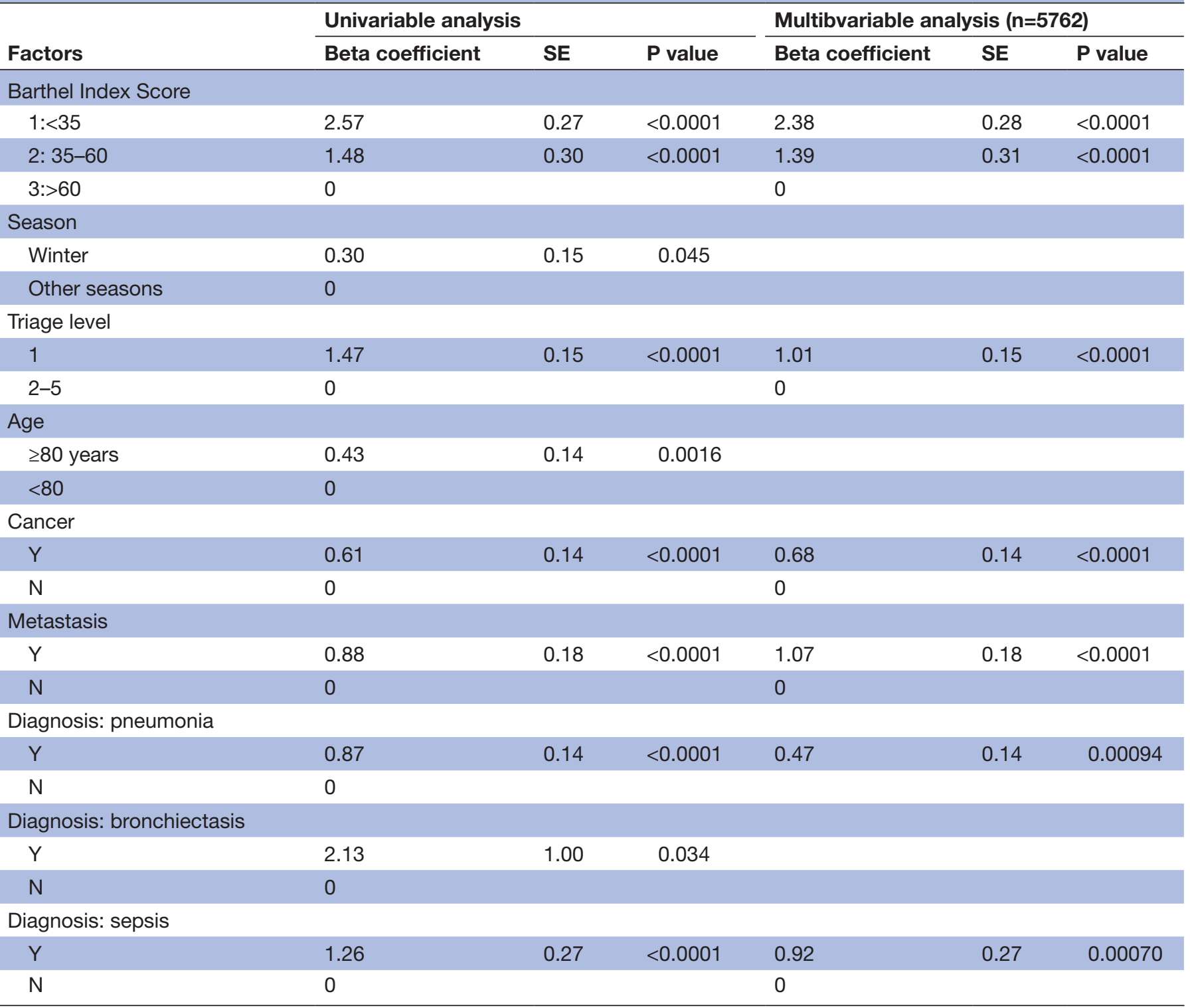


(A) Derivation cohort

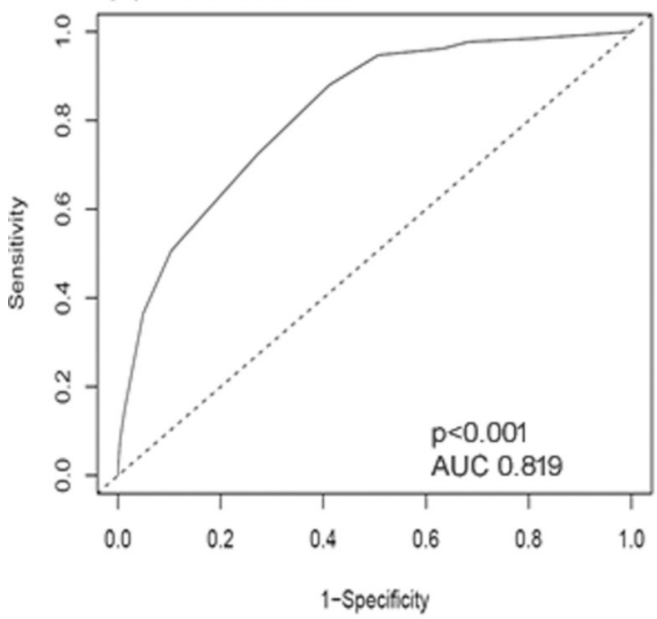

(B) Validation cohort

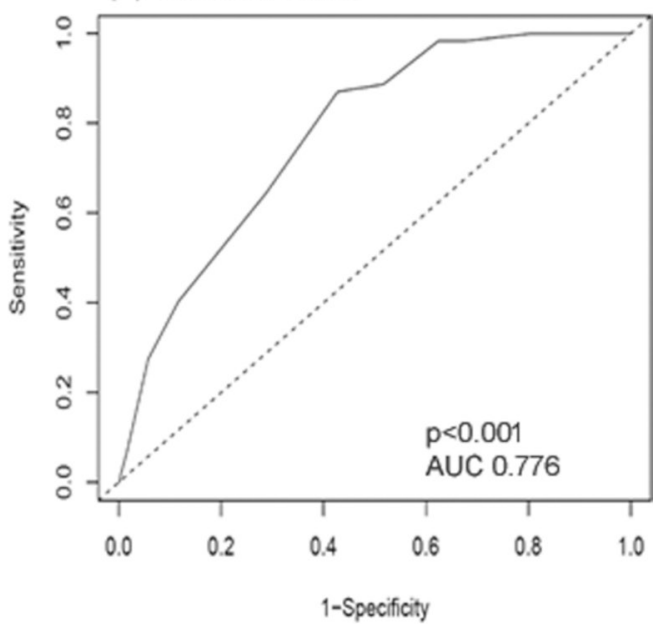

Figure 2 Receiver operating characteristic curves to predict 7-day in-hospital mortality by probability developed from the multivariable model. AUC, area under curve.

\section{DISCUSSION}

This present study found that the 7-day in-hospital mortality was $2.80 \%$ among medical patients admitted from the ED. We established a mortality prediction score at the initial admission using the Barthel Index Score, presence of cancer and metastasis, admission diagnoses of pneumonia and sepsis, and triage level on arrival at the ED. Scores of $\geq 4$ had NPVs of up to $99 \%$ and could be used for exclusion screening. By contrast, scores of $\geq 6$ had specificity of $89 \%$ and PPV of $10.55 \%$, which could cover most of the frail patients.

To manage the mortality outcomes of patients admitted to general medical wards is never easy for hospitalists, although some mortality cannot be avoided. In particular, for patients admitted for unplanned causes leading to ED visits, it is difficult to stratify those who have a high risk for potential death at initial admission. Although prediction scores of NEWS ${ }^{7}$ or clinical alert system ${ }^{11}$ have been developed, the indications of the two scores are mostly regarding the change of vital signs and ensuing critical status that is not strange to initiate intensive care. However, to predict the targeted risk group before they exhibit unstable vital signs is important, for it would allow us to prepare further discussions regarding intensive care and prognosis explanations. By using the prediction score developed and validated in the present study with a large-scale sample, we could stratify the patients easily into three subgroups: low risk for prediction scores of $<4$, intermediate risk for scores of 4-6, and high risk for scores of $\geq 6$. Those with scores of $<4$ can be excluded from the alarm status, and those with scores of $\geq 6$ probably need intensive treatment.

The prediction score showed a high AUROC of 0.819 and a hazard ratio of 1.659 (95\% CI 1.55 to 1.76 per 1 point increment) in the derivation cohort to predict in-hospital mortality within 7 days; the results were similar in the validation group. This score is the first mortality prediction score developed from general medical hospitalised patients and could be applied broadly. However, because the score element is relatively non-specific to diseases, the sensitivity and specificity are not $>90 \%$. Therefore, the score can be used in clinical practice to detect fragile patients at initial admission, but final judgement must be reserved for inpatient physicians.

Among the prediction model, the Barthel index, which measures performance in activities of daily living, can be used to represent general condition and disease severity, and it is one of the important factors in prognosis prediction. For patients with chronic illness, the Barthel Index result can be affected by patients' frailty ${ }^{12} 13$ and disability,

Table 3 Derivation of the prediction score for 7-day in-hospital mortality (data source: derivation cohort)

\begin{tabular}{lllll}
\hline Risk factors & Beta coefficient & SE & P value & $\begin{array}{l}\text { Point values } \\
\text { (beta x 2) }\end{array}$ \\
\hline Barthel index $<35$ vs $>60$ & 2.38 & 0.28 & $<0.0001$ & 5 \\
\hline Barthel index $35-60$ vs $>60$ & 1.39 & 0.31 & $<0.0001$ & 3 \\
\hline Triage: 1 vs $2-5$ & 1.01 & 0.15 & $<0.0001$ & 2 \\
\hline Cancer: Y vs N & 0.68 & 0.14 & $<0.0001$ & 1 \\
Metastasis: Y vs N & 1.07 & 0.18 & $<0.0001$ & 2 \\
Diagnosis of pneumonia: Y vs N & 0.47 & 0.14 & 0.00094 & 1 \\
Diagnosis of sepsis: Y vs N & 0.92 & 0.27 & 0.00070 & 2 \\
\hline
\end{tabular}




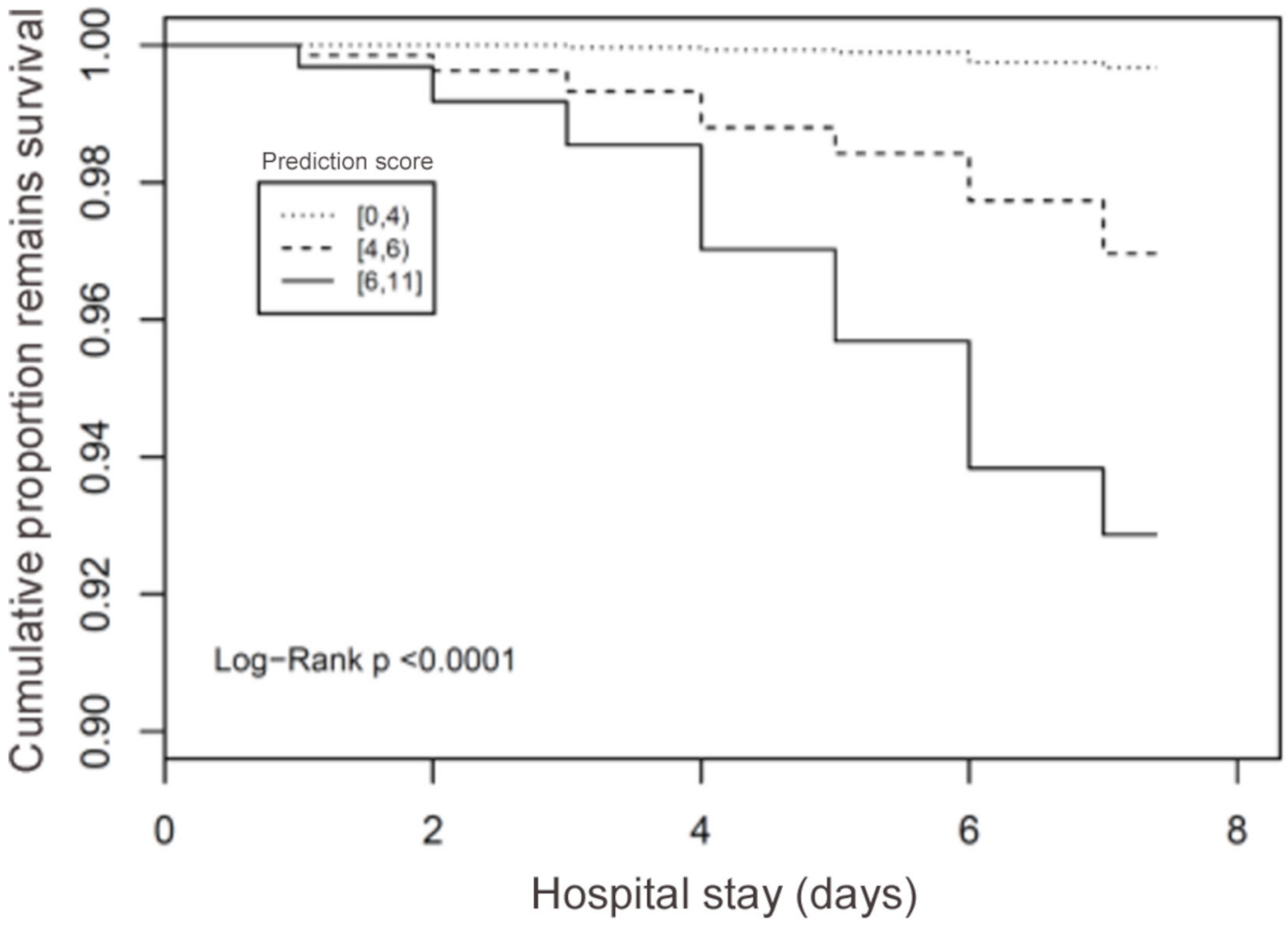

Figure 3 Kaplan-Meier curves for 7 days in-hospital mortality for different ranges of the prediction score.

which correlate with mortality. ${ }^{14}$ On the other hand, it could be the severity of the acute illness which is responsible for the admission. Although we did not discriminate the influence proportions of the Barthel index by acute or chronic illness, it affected the 7-day mortality, with the highest beta coefficient of 2.38 in the multivariable analysis. It might be more easily applied generally at initial admission.

The presence of underlying active cancer and metastatic status are both important predictors for in-hospital mortality due to their immune-compromised status. ${ }^{15}$ The effect of cancer on a patient's outcome has been proven in critical care ${ }^{16}{ }^{17}$ However, whether patients have cancer with or without metastasis may provide little insight on the poor prognosis, and as high as $25 \%$ of terminal cancer patients receive vasopressors in the dying process. $^{18}$ Therefore, hospitalists need to hold family meetings to explain and discuss treatment plans for shared decision making on cancer patients with high prediction scores. ${ }^{19}$

In contrast to a chronic illness such as cancer, triage on arrival to ED could be used as an initial summary index for acute status. The triage index is the five-level TTAS computerised system implemented nationally since $2010 .^{20}$ The triage index includes changes in vital signs, organ failure and acute problems needing immediate treatment. ${ }^{21}$ Triage level 1 at the ED has been classified as an impact factor in the prediction score in the present study. In addition, admission diagnoses including sepsis and pneumonia are responsible for acute illness and associated with 7-day in-hospital mortality. Sepsis is a highmortality syndrome caused by severe infection with or without organ failure. In-hospital mortality could be as high 17\% for patients with sepsis and $26 \%$ for those with severe sepsis. ${ }^{22}$ Both factors (triage at the ED and admission diagnosis) represent acute changes for admitted patients. However, age was statistically significant only in the univariable analysis and not significant in the multivariable Cox analysis, possibly because its effect was erased by other comorbidity and performance statuses.

This study had several limitations. First, we did not record initial vital signs, laboratory results in the original study design of clinical analysis. However, we collected the triage level at ED arrival of each participant, which was associated with initial vital signs. Second, prehospital changes in the medical condition or Barthel Index were not recorded, so their roles in in-hospital mortality prediction require further study. In addition, patients enrolled in this study may have been more severely ill, and a higher proportion may have had cancer, because the present study was conducted in a tertiary referral centre. Third, we only enrolled patients admitted in the hospitalist ward and this study was performed in Taiwan. Therefore, our model may not be generalised to specialty ward and hospitals in other areas before validation. Fourth, the results of this study may be not applicable in hospitals with different triage scales.

\section{CONCLUSION}

The prediction score includes six factors: low Barthel Index Score, triage level 1 at the ED, admission diagnosis of sepsis, diagnosis of pneumonia, and presence of cancer and metastasis. It was developed to screen out the high-risk subgroup for 7-day mortality among patients 
admitted from the ED. Using the prediction score is important and convenient. A score of $<4$ could exclude the alarm status, whereas a score of $\geq 6$ represents a high potential for short-term in-hospital mortality. Further validation is required before generalisation.

\section{Author affiliations}

'Department of Emergency Medicine, National Taiwan University Hospital, Taipei, Taiwan

${ }^{2}$ Division of Hospital Medicine, Department of Internal Medicine, National Taiwan University Hospital, Taipei, Taiwan

${ }^{3}$ Department of Internal Medicine, National Taiwan University College of Medicine, Taipei, Taiwan

${ }^{4}$ Department of Emergency Medicine, National Taiwan University College of Medicine, Taipei, Taiwan

${ }^{5}$ Department of Emergency Medicine, National Taiwan University Hospital, Yunlin Branch, Douliu, Taiwan

\section{Twitter Wen-Chu Chiang @Brian@DrBrian_Tw}

Acknowledgements The authors thank, Department of Medical Research, National Taiwan University Hospital, for their support with data and statistics.

\section{Collaborators N/A}

Contributors C-CS, M-JH and N-CH designed and performed the study. C-CS, $\mathrm{M}-\mathrm{JH}, \mathrm{N}-\mathrm{CH}, \mathrm{Y}-\mathrm{F}, \mathrm{W}-\mathrm{CC}, \mathrm{MH}-\mathrm{MM}$ and $\mathrm{W}-\mathrm{HS}$ were involved in data interpretation, analysis and manuscript preparation. C-CS was responsible for coordinating the study.

Funding The authors have not declared a specific grant for this research from any funding agency in the public, commercial or not-for-profit sectors.

Competing interests None declared.

Patient consent for publication Not required.

Ethics approval The Research Ethics Committee of National Taiwan University Hospital approved this study (IRB No: 201105036RB and 201112161RINC).

Provenance and peer review Not commissioned; externally peer reviewed.

Data availability statement All data relevant to the study are included in the article or uploaded as online supplemental information.

Supplemental material This content has been supplied by the author(s). It has not been vetted by BMJ Publishing Group Limited (BMJ) and may not have been peer-reviewed. Any opinions or recommendations discussed are solely those of the author(s) and are not endorsed by BMJ. BMJ disclaims all liability and responsibility arising from any reliance placed on the content. Where the content includes any translated material, BMJ does not warrant the accuracy and reliability of the translations (including but not limited to local regulations, clinical guidelines, terminology, drug names and drug dosages), and is not responsible for any error and/or omissions arising from translation and adaptation or otherwise.

Open access This is an open access article distributed in accordance with the Creative Commons Attribution Non Commercial (CC BY-NC 4.0) license, which permits others to distribute, remix, adapt, build upon this work noncommercially, and license their derivative works on different terms, provided the original work is properly cited, appropriate credit is given, any changes made indicated, and the use is non-commercial. See: http://creativecommons.org/ licenses/by-nc/4.0/.

\section{ORCID iDs}

Nin-Chieh Hsu http://orcid.org/0000-0002-4997-1081

Chin-Chung Shu http://orcid.org/0000-0003-0311-5148

\section{REFERENCES}

1 Lindenauer PK, Rothberg MB, Pekow PS, et al. Outcomes of care by hospitalists, general internists, and family physicians. N Engl J Med 2007:357:2589-600.

2 Bell CM, Redelmeier DA. Mortality among patients admitted to hospitals on weekends as compared with weekdays. N Engl J Med 2001;345:663-8.

3 Aldridge C, Bion J, Boyal A, et al. Weekend specialist intensity and admission mortality in acute Hospital trusts in England: a crosssectional study. Lancet 2016;388:178-86.

4 Conway R, Cournane S, Byrne D, et al. Time patterns in mortality after an emergency medical admission; relationship to weekday or weekend admission. Eur J Intern Med 2016;36:44-9.

5 Walker AS, Mason A, Quan TP, et al. Mortality risks associated with emergency admissions during weekends and public holidays: an analysis of electronic health records. Lancet 2017;390:62-72.

6 Shiue I, McMeekin P, Price C. Retrospective observational study of emergency admission, readmission and the 'weekend effect'. BMJ Open 2017;7:e012493.

7 Smith GB, Prytherch DR, Meredith P, et al. The ability of the National early warning score (news) to discriminate patients at risk of early cardiac arrest, unanticipated intensive care unit admission, and death. Resuscitation 2013;84:465-70.

8 Hsu N-C, Huang C-C, Shu C-C, et al. Implementation of a sevenday hospitalist program to improve the outcomes of the weekend admission: a retrospective before-after study in Taiwan. PLoS One 2018;13:e0194833.

9 Shu C-C, Lin J-W, Lin Y-F, et al. Evaluating the performance of a hospitalist system in Taiwan: a pioneer study for nationwide health insurance in Asia. $J$ Hosp Med 2011;6:378-82.

$10 \mathrm{Ng}$ C-J, Yen Z-S, Tsai JC-H, et al. Validation of the Taiwan triage and acuity scale: a new computerised five-level triage system. Emerg Med J 2011;28:1026-31.

11 Jerng J-S, Ko W-J, Lu FL, et al. Incidence and significance of clinically abnormal events in a tertiary referral medical center: implementation of the clinical alert system (Cas). J Formos Med Assoc 2008;107:396-403.

12 de la Rica-Escuín M, González-Vaca J, Varela-Pérez R, et al. Frailty and mortality or incident disability in institutionalized older adults: the final study. Maturitas 2014;78:329-34.

13 Goeteyn J, Evans LA, De Cleyn S, et al. Frailty as a predictor of mortality in the elderly emergency general surgery patient. Acta Chir Belg 2017;117:370-5.

14 Pongiglione B, De Stavola BL, Kuper $\mathrm{H}$, et al. Disability and allcause mortality in the older population: evidence from the English longitudinal study of ageing. Eur J Epidemiol 2016;31:735-46.

15 Fiorin de Vasconcellos V, Rcc Bonadio R, Avanço G, et al. Inpatient palliative chemotherapy is associated with high mortality and aggressive end-of-life care in patients with advanced solid tumors and poor performance status. BMC Palliat Care 2019;18:42.

16 Hsiue EH-C, Lee P-L, Chen Y-H, et al. Weaning outcome of solid cancer patients requiring mechanical ventilation in the intensive care unit. J Formos Med Assoc 2019;118:995-1004.

17 Keng L-T, Chung K-P, Lin S-Y, et al. Significant clinical factors associated with long-term mortality in critical cancer patients requiring prolonged mechanical ventilation. Sci Rep 2017;7:2148.

18 Chou H-C, Huang H-L, Chen C-Y, et al. Receipt of vasopressors is positively associated with the length of the actively dying process in hospitalization. Am J Hosp Palliat Care 2018;35:1043-9.

19 Blankenburg R, Hilton JF, Yuan P, et al. Shared decision-making during inpatient rounds: opportunities for improvement in patient engagement and communication. J Hosp Med 2018;13:453-61.

20 Chang W, Liu H-E, Goopy S, et al. Using the five-level Taiwan triage and acuity scale computerized system: factors in decision making by emergency department triage nurses. Clin Nurs Res 2017;26:651-66.

21 Tsai L-H, Huang C-H, Su Y-C, et al. Comparison of prehospital triage and five-level triage system at the emergency department. Emerg Med J 2017;34:720-5.

22 Fleischmann C, Scherag A, Adhikari NKJ, et al. Assessment of global incidence and mortality of Hospital-treated sepsis. current estimates and limitations. Am J Respir Crit Care Med 2016;193:259-72. 